\title{
The role of MRI with diffusion-weighted imaging in restaging rectal cancers after neoadjuvant chemoradiotherapy
}

\begin{tabular}{|c|c|}
\hline \multicolumn{2}{|l|}{$\begin{array}{l}\text { Authors: } \\
\text { Irem Bayram }{ }^{1}\end{array}$} \\
\hline \multicolumn{2}{|l|}{ Baris Bakir ${ }^{1}$} \\
\hline \multicolumn{2}{|c|}{ Merve G.D. Kartal ${ }^{1}$} \\
\hline \multicolumn{2}{|l|}{ Enver Kunduz ${ }^{2}$} \\
\hline \multicolumn{2}{|l|}{ Onur Bayram ${ }^{3}$} \\
\hline \multicolumn{2}{|l|}{ Rustu Turkay ${ }^{4}$} \\
\hline \multicolumn{2}{|l|}{ Oktar Asoglu ${ }^{5}$} \\
\hline \multicolumn{2}{|l|}{ Yersu Kapran ${ }^{6}$} \\
\hline \multicolumn{2}{|c|}{$\begin{array}{l}{ }^{1} \text { School of Medicine, Istanbul } \\
\text { University, Turkey }\end{array}$} \\
\hline \multicolumn{2}{|c|}{$\begin{array}{l}{ }^{2} \text { Department of General } \\
\text { Surgery, Bezmi Alem Vakıf } \\
\text { University, Turkey }\end{array}$} \\
\hline \multicolumn{2}{|c|}{$\begin{array}{l}{ }^{3} \text { School of Medicine, Koc } \\
\text { University, Turkey }\end{array}$} \\
\hline \multicolumn{2}{|c|}{$\begin{array}{l}{ }^{4} \text { Bakırköy Dr. Sadi Konuk } \\
\text { Training and Research } \\
\text { Hospital, Istanbul, Turkey }\end{array}$} \\
\hline \multicolumn{2}{|c|}{${ }^{5}$ Liv Hospital, Istanbul, Turkey } \\
\hline \multicolumn{2}{|c|}{$\begin{array}{l}{ }^{6} \text { American Hospital, Istanbul, } \\
\text { Turkey }\end{array}$} \\
\hline \multicolumn{2}{|c|}{ Research Project no.: 16/2014 } \\
\hline \multicolumn{2}{|c|}{$\begin{array}{l}\text { Corresponding author: } \\
\text { Irem Bayram, } \\
\text { dr.irembayram@gmail.com }\end{array}$} \\
\hline \multicolumn{2}{|c|}{$\begin{array}{l}\text { Received: } 08 \text { Dec. } 2015 \\
\text { Accepted: } 18 \text { July } 2016 \\
\text { Published: } 21 \text { Sept. } 2016\end{array}$} \\
\hline \multicolumn{2}{|c|}{$\begin{array}{l}\text { How to cite this article: } \\
\text { Bayram I, Bakir B, Kartal } \\
\text { MGD, et al. The role of MRI } \\
\text { with diffusion-weighted } \\
\text { imaging in restaging rectal } \\
\text { cancers after neoadjuvant } \\
\text { chemoradiotherapy. S Afr J } \\
\text { Rad. 2016;20(1), a967. } \\
\text { http://dx.doi.org/10.4102/ } \\
\text { sajr.v20i1.967 }\end{array}$} \\
\hline \multicolumn{2}{|l|}{ Read online: } \\
\hline 品科回 & $\begin{array}{l}\text { Scan this QR } \\
\text { code with your } \\
\text { smart phone or } \\
\text { mobile device } \\
\text { to read online. }\end{array}$ \\
\hline
\end{tabular}

Background: It is challenging to restage rectal cancer at MRI, in patients who have had neoadjuvant chemoradiotherapy.

Objective: To investigate the accuracy of MRI with diffusion-weighted imaging (DWI) in the restaging of rectal cancer.

Materials and methods: Pre- and post-neoadjuvant chemoradiotherapy MRI examinations of 35 patients diagnosed with locally advanced rectal cancer were evaluated and subsequently compared with post-operative pathology results.

Results: The accuracy of MRI with DWI to determine the T-stage status was calculated as $54.28 \%$. Kappa statistics revealed poor concordance with pathology results, with a $\kappa$ value of $0.212 \pm 0.114(p=0.028)$. The apparent diffusion coefficient (ADC) values measured after the neoadjuvant chemotherapy revealed a significant increase when compared with pre-treatment ADC values $(p<0.000001)$. MRI accuracy rate for lymph node involvement was calculated as $57.14 \%$ with a $\kappa$ value of $0.001(p=0.989)$. MRI had $80 \%$ sensitivity and $100 \%$ specificity in determining mesorectal fascia involvement, with a calculated positive predictive value of $100 \%$ and a calculated negative predictive value of $96 \%$. The accuracy of MRI in overall staging according to the TNM staging system was $28 \%$.

Conclusion: The accuracy of MRI in restaging rectal cancer is not yet sufficient and is not on par with the accuracy of MRI in the primary staging of the disease. This is attributed to post-treatment changes. Adding DWI to the protocol is promising, but more expanded data are required.

\section{Introduction}

Magnetic resonance imaging (MRI) has been shown to be a useful tool in staging rectal cancer preoperatively. ${ }^{1,2,3,4,5}$ It has been reported to be highly accurate in the detection of circumferential resection margin (CRM) involvement and defining the T-stage of the tumour, but assessment of lymph node involvement is not as successful. In a recent meta-analysis, MRI specificity for CRM involvement was $95 \%$, for T-stage determination $75 \%$, and for the detection of lymph node involvement $75 \% .^{5}$

Accurate restaging after neoadjuvant therapy provides an option to modify the initial plan of surgery. An optimal tool to use in restaging should accurately assess if the tumour has downsized to a lower stage tumour and if the tumour involvement of the CRM and other organs has regressed, so that total mesorectal excision (TME) can be performed.

Diffusion-weighted MRI is a functional MRI technique that is based on differences in the extracellular movement of water protons which is determined by the cellularity of the structures. In tissues with normal cellularity, water protons diffuse freely, and they are appreciated as low signal structures on diffusion-weighted imaging (DWI). On the other hand, in tissues with increased cellularity such as tumour tissues, the diffusion of water is restricted, resulting in remaining high signal on DWI. DWI has been shown as a promising tool for the identification of malignant tumours, and recent studies on rectal cancer have indicated that DWI may be useful for response evaluation after chemoradiation treatment. In 2009, Kim et al. showed in a study on 40 patients that DWI in addition to standard MRI significantly improved the performance of radiologists in selecting complete responders compared with standard MRI only. ${ }^{6}$

Copyright: (C) 2016. The Authors. Licensee: AOSIS. This work is licensed under the Creative Commons Attribution License. 
In the present study, our aim was to investigate the accuracy of MRI with DWI in the restaging of rectal cancer. Whilst the performance of MRI in the determination of the presence of the prognostic parameters is assessed separately, accuracy in detection of the overall stage of the tumour is also identified.

\section{Materials and methods}

The patients diagnosed with mid- and distally located rectal tumour, staged as locally advanced (T3 and/or N+) and treated with long-term neoadjuvant therapy (fractioned 45 Gy radiotherapy and 5-flourouracil + folinic acid chemotherapy) in our hospital, between the years 2010 and 2013, were retrospectively evaluated. A total of 35 patients (10 women (28.5\%) and 25 men $(71.5 \%)$ ) who ranged from 27 to 76 years old (mean 55.9 years) had undergone pelvic MRI prior to-treatment and in the 7th or 8th week of the treatment. Of these, 23 patients $(65.7 \%)$ had distally located tumours and 12 patients $(34.3 \%$ ) had tumours located at midrectal level.

The pathology results of these patients were retrospectively evaluated and compared. Blinded to the pathology results, the tumour T-stages (before and after the neoadjuvant therapy), CRM positivities, changes in diffusion signal intensity, apparent diffusion coefficient (ADC) values and presence of positive lymph nodes were assessed with MRI. In pre- and post-treatment MRIs, the T-stage of the tumour, diffusion signal intensity and changes in ADC values were compared. T-stages, accuracy of the presence of pathological lymph nodes and accuracy of CRM positivities, determined by post-treatment MRIs, were compared with the results of post-operative pathology results.

\section{MRI protocol}

The scans were performed with a 1.5 Tesla MR (Symphony Siemens, Erlangen, Germany) scanner with a body coil activated in a spinal coil. Firstly, T2-weighted (W) turbo spin-echo (TSE) axial sections were acquired, from the pelvic oriented iliac artery bifurcation level up to the end of the anal canal. After localising the tumour in the pelvic oriented coronal and sagittal T2-W TSE sections, tumour oriented thin-section ( $3 \mathrm{~mm}$ ) axial T1-W TSE and axial T1-W TSE fat saturation (FS) sections were obtained. Subsequently, post contrast $\mathrm{T} 1-\mathrm{W}$ sequences were acquired in the axial and saggital planes. Additionally, a DWI with tumour region orientation was performed in the axial plane. The parameters used are explained in detail in Table 1.

\section{Evaluation of images}

Previously reported MRI examinations were re-evaluated by two radiologists, one with 10 years' experience in pelvic MRI and the other with 4 years' experience. Both were blinded to previous reports and pathology results in consensus.

Tumour localisation was determined on the sagittal T2-W series on both the pre- and post-contrast MR examinations, and they were grouped as distal $(0 \mathrm{~cm}-5 \mathrm{~cm})$, middle $(5 \mathrm{~cm}-10 \mathrm{~cm})$ and proximal $(10 \mathrm{~cm}-15 \mathrm{~cm})$ with respect to localisation.

TNM staging criteria were used for staging rectal tumours. $\mathrm{T}$ - and $\mathrm{N}$ - staging was performed. To determine the T-stage status on post-contrast MRI, a solid mass with intermediate signal intensity on T2-W series was considered a definite criterion. Hypointense signal intensity changes indicated fibrosis. On the diffusion images, residual high-signal intensity on the location of the primary tumour was considered a criterion for residual tumour, whereas the absence of increased signal on DWI was indicative of a complete response. Stage T1 and T2 tumours were grouped together. Tumours that extended through the muscle layer to the perirectal fat tissue were T3, and tumours that had reached the adjacent organs were $\mathrm{T} 4$.

Perirectal and iliac lymph nodes $\geq 5 \mathrm{~mm}$ in diameter and with irregular margins were defined as metastatic. The total number of pathologic lymph nodes and the dimension of the biggest lymph node were recorded for each patient.

Pre- and post-contrast tumour was evaluated on diffusionweighted MRI images obtained by b1000. They were grouped as 'isointense-hypointense' or hyperintense, relative to the normal rectal wall. The ADC values of these regions on the ADC map views were quantitatively recorded.

The presence of mesorectal fascia (MRF) invasion (CRM (+)) was noted; and it was defined as extension of the tumour itself to the MRF or presence of an implant or an abnormal lymph node at a distance of $<1 \mathrm{~mm}$.

SPSS version 10.0 (SPSS, Cary, USA) was used for statistical analysis. Kappa statistics were used to establish correlation

TABLE 1: Parameters used in both pre- and post-contrast (C) MRI examinations.

\begin{tabular}{|c|c|c|c|c|c|c|c|}
\hline Sequence & $\begin{array}{l}\text { Time of repetition } \\
\text { (TR) (ms) }\end{array}$ & $\begin{array}{l}\text { Time of echo (TE) } \\
(\mathrm{ms})\end{array}$ & Average & Slice thickness (mm) & Slice number & Voxel size (mm) & FOV $(\mathrm{mm})$ \\
\hline T2 TSE sag & 3620 & 130 & 3 & 3 & 20 & $0.8 \times 0.6 \times 3.0$ & 220 \\
\hline T2 TSE cor & 7500 & 115 & 3 & 3 & 30 & $1.2 \times 0.8 \times 3.0$ & 300 \\
\hline T2 TSE ax & 4430 & 134 & 3 & 3 & 25 & $0.8 \times 0.6 \times 3.0$ & 180 \\
\hline T1 TSE ax & 525 & 12 & 2 & 3 & 25 & $0.7 \times 0.5 \times 3.0$ & 180 \\
\hline T1 TSE FS ax & 520 & 12 & 2 & 3 & 25 & $0.8 \times 0.6 \times 3.0$ & 180 \\
\hline DWI ax & 4400 & 86 & 4 & 5 & 20 & $2.1 \times 2.1 \times 5.0$ & 400 \\
\hline T1 TSE FS ax post $C$ & 520 & 12 & 2 & 3 & 25 & $0.7 \times 0.5 \times 3.0$ & 180 \\
\hline T1 TSE FS sag post $C$ & 400 & 12 & 2 & 3 & 20 & $1.0 \times 0.7 \times 3.0$ & 220 \\
\hline
\end{tabular}


between MRI and pathology results in determining the $\mathrm{T}$ and $\mathrm{N}$ stages. The paired $t$-test was used to establish differences between ADC values.

\section{Results}

The T-stage distribution as evaluated by pre- and postneoadjuvant therapy MRI examinations, is presented in Table 2 .

\section{T stage}

After neoadjuvant therapy, in 32 of 35 patients (91.4\%), tumour signal intensity changed from hyperintense to hypointense, indicating that the diffusion restriction had resolved. On the contrary, in three patients $(8.6 \%)$ a posttreatment diffusion restriction in the tumour lesion site persisted. Post-treatment MRI examinations of all three of these patients were interpreted as tumour positive, but tumour cells were not found in any of their pathology specimens. Of the 32 patients with no restricted diffusion, 21 had residual tumour and 11 had a complete response according to the pathology results. Comparison of postneoadjuvant therapy T-staging and pathology T-staging is presented in Table 3.

The accuracy of MRI with DWI to determine the T-stage status was calculated at $54.28 \%$. Kappa statistics revealed a poor concordance with pathology results $(\kappa$ value $0.212 \pm$ $0.114(p=0.028))$.

The ADC values calculated after the neoadjuvant treatment revealed a significant increase when compared with pretreatment ADC values $(p<0.000001)$. Details appear in Table 4 .

\section{N stage}

When pre- and post-neoadjuvant therapy lymph node involvement data were compared, a statistically significant regression was observed $(p<0.0001)$. Whilst before treatment,

TABLE 2: T-Stage distributions detected by pre- and post-neoadjuvant therapy MRI examinations.

\begin{tabular}{lccc}
\hline T-stage distribution & Tumour T stage & $\boldsymbol{n}$ & $\mathbf{\%}$ \\
\hline Before neoadjuvant & T2 & 1 & 2.8 \\
therapy & T3 & 33 & 94.4 \\
& T4 & 1 & 2.8 \\
After neoadjuvant & T0 & 3 & 8.5 \\
therapy & T2 & 5 & 14.2 \\
& T3 & 26 & 74.2 \\
& T4 & 1 & 2.8 \\
\hline
\end{tabular}

$\mathrm{N}$-stage status was positive in 33 patients, this number was found to be 15 post treatment.

Lymph node status was positive in nine patients at MRI but their pathology results reported no observed metastatic lymph nodes. When categorised, the biggest lymph node dimensions on MRI for all these patients were between $5 \mathrm{~mm}$ and $8 \mathrm{~mm}$. In five of these patients the biggest lymph nodes had minimal irregularity at their borders. The biggest dissected lymph nodes of these patients were measured and categorised at pathology; two of them were $2 \mathrm{~mm}-5 \mathrm{~mm}$, five were $5 \mathrm{~mm}-8 \mathrm{~mm}$, and two were $>8 \mathrm{~mm}$.

Six patients were lymph node negative at MRI, but their pathology demonstrated metastatic lymph nodes. When the biggest lymph nodes were measured at MRI, these six patients were categorised by their dimensions: two were $1 \mathrm{~mm}-2 \mathrm{~mm}$, and four were $2 \mathrm{~mm}-5 \mathrm{~mm}$; all of these lymph nodes were morphologically categorised as benign. After dissection of the specimens, the biggest lymph nodes at pathology were between $5 \mathrm{~mm}$ and $8 \mathrm{~mm}$.

In the 12 patients with pathologically proven lymph node involvement, the biggest lymph nodes at MRI were categorised based on their dimensions: four were $1 \mathrm{~mm}-2 \mathrm{~mm}$, two were $2 \mathrm{~mm}-5 \mathrm{~mm}$, four were $5 \mathrm{~mm}-8 \mathrm{~mm}$, and two were $>8 \mathrm{~mm}$. The biggest dissected lymph nodes in these patients were measured at pathology: 10 were $5 \mathrm{~mm}-8 \mathrm{~mm}$ and 2 were $>8 \mathrm{~mm}$.

Amongst the 23 patients who had negative pathology results for lymph node involvement, the biggest lymph node dimensions observed at MRI were $1 \mathrm{~mm}-2 \mathrm{~mm}$ for 2 patients, $2 \mathrm{~mm}-5 \mathrm{~mm}$ for 13 patients, and $5 \mathrm{~mm}-8 \mathrm{~mm}$ for 8 patients. When the dimensions of the biggest dissected lymph nodes in the pathology reports were categorised, 3 of them were $2 \mathrm{~mm}-5 \mathrm{~mm}, 14$ were $5 \mathrm{~mm}-8 \mathrm{~mm}$, and 6 were $>8 \mathrm{~mm}$.

The overall N-stage status of the patients is set out in Table 5. The MRI accuracy rate for lymph node involvement was calculated at $57.14 \%$ with a $\kappa$ value of $0.001(p=0.989)$.

TABLE 4: Comparison of tumour ADC values before and after neoadjuvant therapy.

\begin{tabular}{lcc}
\hline Variable & $\begin{array}{c}\text { ADC before neoadjuvant } \\
\text { therapy }\end{array}$ & $\begin{array}{c}\text { ADC after neoadjuvant } \\
\text { therapy }\end{array}$ \\
\hline Number of patients & 35 & 35 \\
Average & 0.920 & 1.34 \\
Minimum value & 0.57 & 0.9 \\
Maximum value & 1.50 & 2.2 \\
\hline
\end{tabular}

TABLE 3: Comparison of post-neoadjuvant therapy T-staging and pathology T-staging.

\begin{tabular}{lcccc}
\hline $\begin{array}{l}\text { MRI T-stage after } \\
\text { neoadjuvant therapy }\end{array}$ & \multicolumn{3}{c}{ Post-operative pathology T stage } \\
\cline { 2 - 5 } & T0 & T1 & T2 & T3 \\
\hline T0 & 2 & 0 & 0 & 1 \\
T1 & 0 & 0 & 0 & 0 \\
T2 & 2 & 0 & 2 & 0 \\
T3 & 7 & 2 & 1 & 15 \\
T4 & 0 & 0 & 0 & 0 \\
\hline
\end{tabular}




\section{CRM status}

Prior to neoadjuvant therapy, the MRF involvement of 27 patients was reported as negative and the MRF involvement of eight patients was positive, whereas after the therapy, the number of patients with negative MRF involvement increased to 30 and the number of patients with positive MRF involvement decreased to 5 . As per postoperative pathology reports, 31 patients had negative and four patients had positive MRF involvements. According to this data, MRI had an $80 \%$ sensitivity and $100 \%$ specificity in determining MRF involvement, with a calculated positive predictive value of $100 \%$ and a negative predictive value (NPV) of $96 \%$.

\section{TNM staging}

According to post-neoadjuvant therapy MRI scan staging, 3 patients were Stage 0, 5 patients were Stage 1, 13 patients were Stage $2 \mathrm{a}$ and 14 patients were Stage $3 \mathrm{~b}$. The postoperative pathology staging is summarised in Table 6. The results show, according to the TNM staging system, that in a total of 35 patients, 13 cases $(37.14 \%$ ) were overstaged (Figure 1a and b), 3 cases $(8.57 \%)$ were downstaged (Figure 2) and 19 cases (54.28\%) were correctly staged (Figure 3). Reasons for overstaging include fibrosis, desmoplastic reaction and lymphoplasmocytic infiltration. A reason for downstaging may be that MRI cannot differentiate tumour tissue. The accuracy of MRI in the overall staging according to the TNM staging system was $28 \%$.

\section{Discussion}

Traditionally, rectal cancer staging was done histopathologically, after surgery, to achieve a prognosis. However, with the implementation of adjuvant therapy, staging has become more relevant to the available therapeutic options. Preoperative staging is now crucial as the surgical techniques have evolved and neoadjuvant therapy has been implemented as an alternative in the treatment protocol. This advancement has also raised the issue of restaging before surgery in order to modify treatment strategy according to tumour response.
What is expected from MRI as an imaging tool in the determination of treatment response and disease restaging, is determination of the depth of invasion within the rectal wall which determines the $\mathrm{T}$ stage, detecting lymph node involvement which determines the $\mathrm{N}$ stage, and examining the involvement or the distance to MRF which determines the CRM status.

A new 'wait and see' approach has recently been debated in the management of complete responders. ${ }^{7,9}$ This requires a careful selection of patients with a complete response. In the present study, three patients were determined to be complete
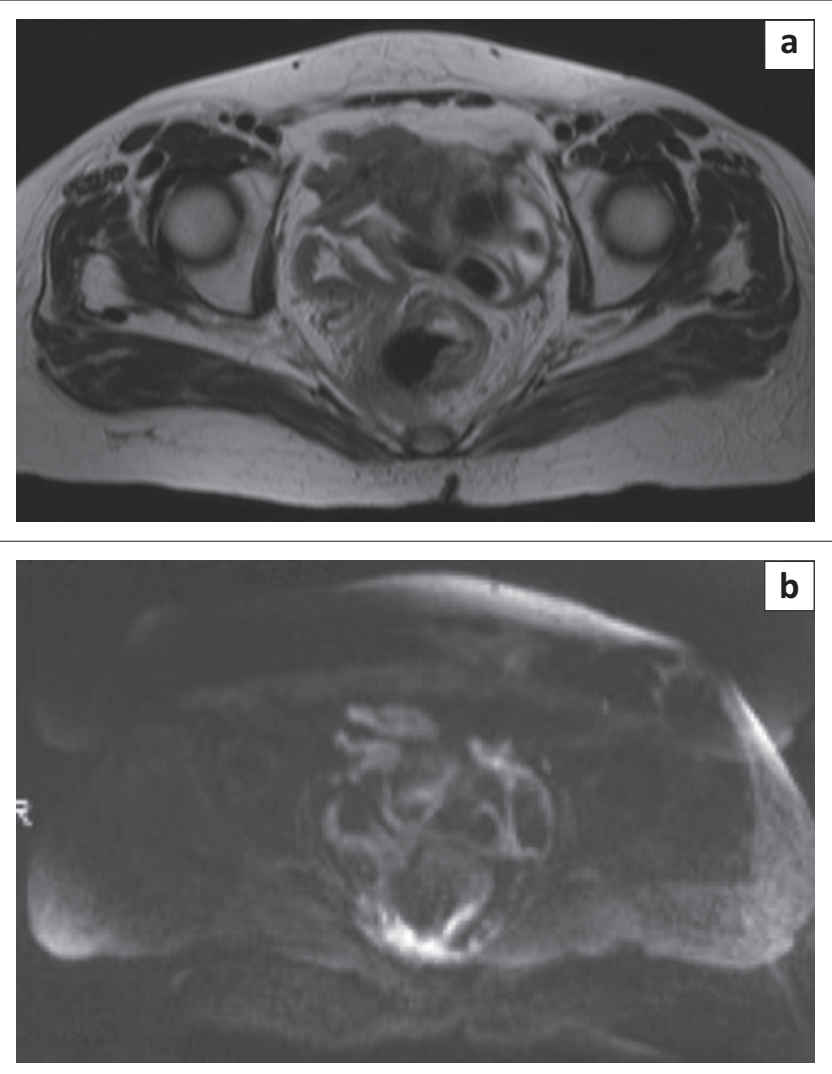

Note: The tumour tissue was staged as T3 at MRI but the pathology staging was T0.

FIGURE 1: After neoadjuvant therapy, there is residual tumour tissue seen at $6-9$ o'clock on the T2 axial sequence (a) with restriction in the tumoural area on the diffusion-weighted sequence $(b)$.

TABLE 5: Comparison of post-neoadjuvant therapy MRIs and pathological N-stage.

\begin{tabular}{|c|c|c|c|c|c|}
\hline \multirow{2}{*}{$\begin{array}{l}\mathrm{N} \text {-staging at MRI after } \\
\text { neoadjuvant therapy }\end{array}$} & \multicolumn{5}{|c|}{ Post-operative pathology $N$ stage } \\
\hline & 0 & 1a & $1 \mathrm{~b}$ & $2 a$ & $2 b$ \\
\hline 0 & 14 & 5 & 0 & 1 & 0 \\
\hline 1a & 5 & 0 & 1 & 0 & 1 \\
\hline $1 b$ & 3 & 1 & 0 & 1 & 0 \\
\hline $2 a$ & 1 & 0 & 1 & 0 & 1 \\
\hline
\end{tabular}

TABLE 6: Comparison of post-neoadjuvant therapy MRI tumour stages and the pathology tumour stages.

\begin{tabular}{|c|c|c|c|c|c|c|c|}
\hline \multirow{2}{*}{$\begin{array}{l}\text { MRI staging after } \\
\text { neoadjuvant therapy }\end{array}$} & \multicolumn{7}{|c|}{ Post-operative pathology stage } \\
\hline & Stage 0 & Stage 1 & Stage 2a & Stage $\mathbf{2 b}$ & Stage 3a & Stage $\mathbf{3 b}$ & Stage $3 c$ \\
\hline Stage 0 & 2 & 0 & 0 & 0 & 0 & 1 & 0 \\
\hline Stage 1 & 3 & 2 & 0 & 0 & 0 & 0 & 0 \\
\hline Stage $2 a$ & 5 & 2 & 2 & 0 & 1 & 3 & 0 \\
\hline Stage $3 b$ & 2 & 1 & 5 & 1 & 0 & 4 & 1 \\
\hline
\end{tabular}




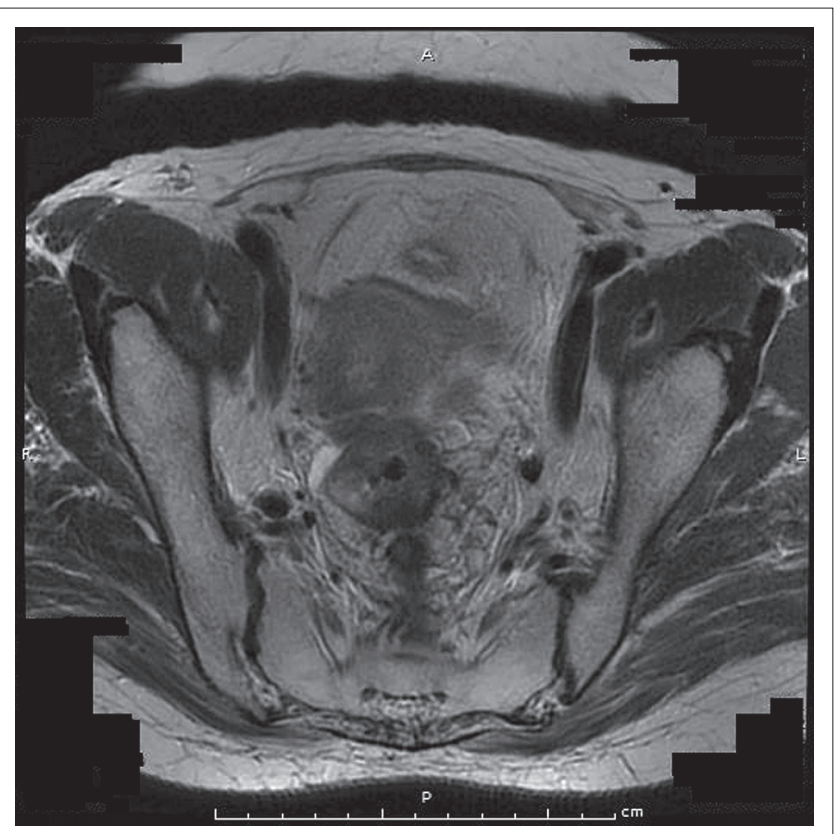

FIGURE 2: After neoadjuvant therapy, the circular wall thickening in the axial T2 sequence was evaluated as Stage T2 tumour at MRI but the pathology result was Stage T4.

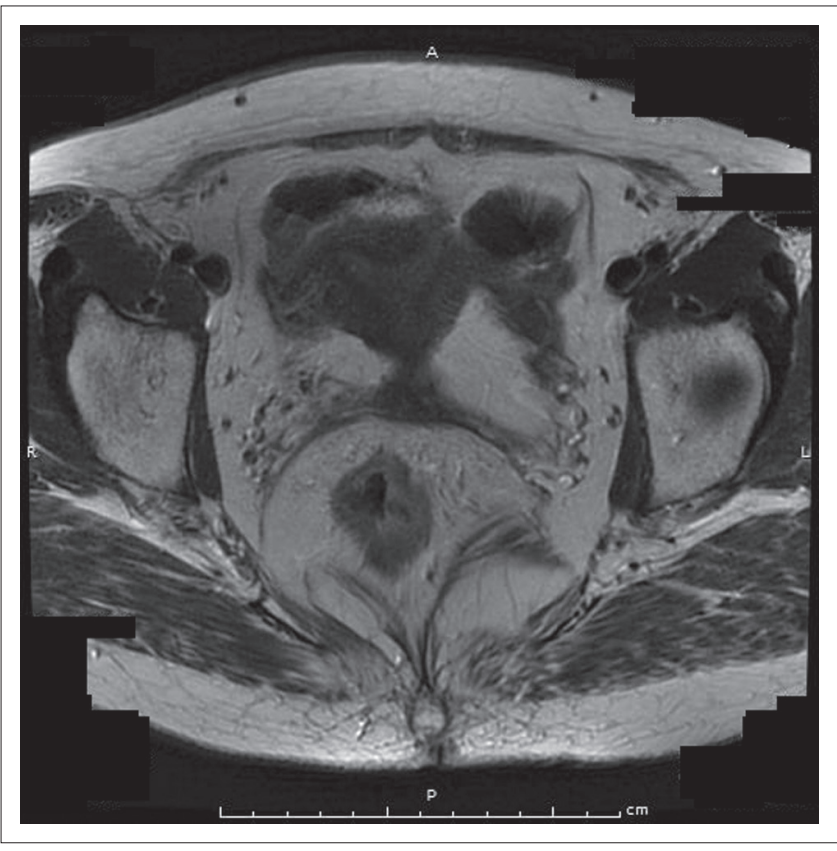

FIGURE 3: In the T2 axial plane, there is residual tumour tissue in the right half and posterior part of the wall (5-10 o'clock) which was reported as Stage T3 at MRI. The pathology results also reported Stage T3.

responders, but pathology revealed that one of these patients had a T3 tumour. Conversely, out of 11 patients who were assigned as complete responders according to the pathology results, only two were determined to be complete responders at MRI, and the rest were overstaged, which would change the management plan dramatically. According to the final pathology reports, the factors attributed to the discrepancy between pathology and MRI examinations may be listed as radiation-induced tumour fibrosis, rectal wall thickening and complex pelvic anatomy. Radiation exposure results in fibrosis of the tumour, oedema of the rectal wall, and a desmoplastic reaction which are hard to distinguish from viable tumour tissue, particularly owing to diffusion restriction also seen with these changes. In the present study, it has been shown that ADC values increase significantly in treated tumours.

The accuracy of MRI is reduced particularly in distinguishing T2-stage tumours from T3, with a specificity of $70 \%$ for T2 and $75 \%$ for T3. ${ }^{2,10}$ In a study by The MRI and Rectal Cancer European Equivalence Study (MERCURY) group, it was explained that T3-stage tumours demonstrate variable prognosis and should be investigated in three subgroups according to the degree of extension of the tumour to the MRF. ${ }^{11}$ In this sense, distinguishing T2 tumours from superficial T3 tumours does not really change the management plan. Although in the current study, stage T2 and T3 tumours were successfully distinguished consistent with pathology results, the number of included patients was small.

In rectal cancer patients, correct assessment of lymph node involvement is considered to be an important prognostic factor. Currently, there is still no general consensus about the dimensions or forms in nodal involvement evaluations.

In a 2004 study by Kim J.H. et al. on 75 patients, the criteria for nodal staging in rectal cancer of accuracy, specificity and sensitivity rates, were examined based on the form and dimension of lymph nodes. ${ }^{12}$ Lymph nodes bigger than $5 \mathrm{~mm}$ were defined as positive and the accuracy rate was reported as $75 \%$, sensitivity $73 \%$ and specificity $75 \%$, with a positive predictive value of $55 \%$. When defining positive as $>3 \mathrm{~mm}$, the rates would be $57 \%, 91 \%, 53 \%$ and $40 \%$ respectively; and above $10 \mathrm{~mm}$ would be $81 \%, 36 \%, 100 \%$ and $100 \%$ respectively. At the same time, the effects of lymph node characteristics such as form, contour, signal intensity or heterogeneity on lymph node positivity should be further examined. Considering lymph node contours, spiculation and irregular contour were statistically meaningful in determining tumour involvement.

In another study where CT (computed tomography), MRI and ERUS (endorectal ultrasound) were compared in nodal staging assessment, accuracy rates (with a cut-off value of $5 \mathrm{~mm}$ ) were reported as $62 \%, 68 \%$ and $65 \%$ respectively, and negative predictive values (NPVs) were reported as $88 \%$, $78 \%$ and $76 \%$ respectively. ${ }^{13}$

CRM involvement is also considered a strong predictor for recurrence in rectal cancer patients. ${ }^{13}$ MRI can provide accurate information in this regard at a rate as high as $85 \% .{ }^{14}$

In the current study, the overall TNM staging of the tumours was determined and compared with the pathology results. According to the TNM staging system, the accuracy of MRI was $28 \%$ which is very low. Acknowledging the fact that the performance of MRI in the detection of T-stage and N-stage status was not significant, it should be noted that the CRM, which was significant and is a critical prognostic predictor, is not included in the staging system. 
One of the limitations of the study is that DWI was not separately evaluated to demonstrate its added value. However, patients with and without diffusion restriction were grouped separately and compared with pathology reports. Additionally, the data were not reviewed by the two radiologists separately, and inter- and intra-observer variations were not stratified.

\section{Conclusion}

Despite being a useful tool in the primary staging of rectal cancer, MRI does not yet suffice as a tool for restaging. On the other hand, because of its success in estimating T3 and lowerstaged tumours, cases with negative lymph nodes and CRMnegative patients, it is still promising in the selection of patients for local excision. Further studies with larger series numbers are needed to demonstrate the feasibility of the technique.

\section{Competing interests}

The authors declare that they have no financial or personal relationships which may have inappropriately influenced them in writing this article.

\section{Authors' contributions}

I.B. was the guarantor of integrity of the entire study. M.K., B.B. and I.B. originated the study concepts and design. E.K. did the literature research, and Y.K. and O.A. were responsible for the clinical studies. M.K. and O.B. were responsible for the experimental studies and/or data analysis, and R.T. for the statistical analysis. I.B. wrote the initial manuscript and B.B. and O.B. were responsible for editing it.

\section{References}

1. Kwok H, Bissett IP, Hill GL. Preoperative staging of rectal cancer. Int J Colorectal Dis. 2000;15:9-20. http://dx.doi.org/10.1007/s003840050002

2. Bipat S, Glas AS, Slors FJM, et al. Rectal cancer: Local staging and assessment of lymph node involvement with endoluminal US, CT, and MR imaging: A meta-analysis. Radiology. 2004;232:773-783. http://dx.doi.org/10.1148/radiol.2323031368

3. Lahaye MJ, Engelen SME, Nelemans PJ, et al. Imaging for predicting the risk factors, the circumferential resection margin and nodal disease, of local
recurrence in rectal cancer: A meta-analysis. Semin Ultrasound CT MR. 2005;26:259-268. http://dx.doi.org/10.1053/j.sult.2005.04.005

4. Purkayastha $\mathrm{S}$, Tekkis PP, Athanasiou T, et al. Diagnostic precision of magnetic resonance imaging for preoperative prediction of the circumferential margin involvement in patients with rectal cancer. Colorectal Dis. 2006;9:402-411. http:// dx.doi.org/10.1111/j.1463-1318.2006.01104.x

5. Al-Sukhni E, Milot L, Fruitman M, et al. Diagnostic accuracy of MRI for assessment of $\mathrm{T}$ category, lymph node metastases, and circumferential resection margin involvement in patients with rectal cancer: A systematic review and meta-analysis. Ann Surg Oncol. 2012;19(7):2212-2223. http://dx.doi.org/10.1245/s10434-0112210-5

6. Kim SH, Lee JM, Hong SH, et al. Locally advanced rectal cancer: Added value of diffusion weighted MR imaging in the evaluation of tumor response to neoadjuvant chemo- and radiation therapy. Radiology. 2009;253:116-125. http:// dx.doi.org/10.1148/radiol.2532090027

7. Habr-Gama A, Perez RO. Non-operative management of rectal cancer after neoadjuvant chemoradiation. Br J Surg 2009;96:125-127. http://dx.doi.org/10.1002/ bjs.6470

8. Habr-Gama A, Perez RO, Nadalin W, et al. Operative versus nonoperative treatment for stage 0 distal rectal cancer following chemoradiation therapy: Long-term results. Ann Surg. 2004; 240: 711-717. http://dx.doi.org/10.1097/01.sla.000014 1194.27992.32

9. Habr-Gama A, Perez R, Proscurshim I, Gama-Rodrigues J. Complete clinical response after neoadjuvant chemoradiation for distal rectal cancer. Surg Oncol Clin N Am. 2010; 19: 829-845. http://dx.doi.org/10.1016/j.soc.2010.08.001

10. Kim DJ, Kim JH, Lim JS, et al. Restaging of rectal cancer with MR imaging after concurrent chemotherapy and radiation therapy. Radiographics. 2010; 30: 503-516. http://dx.doi.org/10.1148/rg.302095046

11. Taylor FG, Quirke P, Heald RJ, et al. Preoperative high-resolution magnetic resonance imaging can identify good prognosis stage I, II, and III rectal cancer best managed by surgery alone: A prospective, multicenter, European study. Ann Surg. 2011;253:711-719. http://dx.doi.org/10.1097/SLA.0b013e31820b8d52

12. Kim JH, Beets GL, Kim MJ, Kessels AG, Beets-Tan RG. High resolution MR imagin for nodal staging in rectal cancer: Are there any criteria in addition to the size? Eur J Radiol. 2004;52:78-83. http://dx.doi.org/10.1016/j.ejrad.2003.12.005

13. Wang C, Zhou Z, Wang Z, et al. Patterns of neoplastic foci and lymph node micrometastasis within the mesorectum. Langenbecks Arch Surg. 2005;390(4): 312-318. http://dx.doi.org/10.1007/s00423-005-0562-7

14. Pomerri F, Pucciarelli S, Maretto I, et al. Prospective assessment of imaging after preoperative chemoradiotherapy for rectal cancer. J Surg. 2011 ;149(1):56-64. http://dx.doi.org/10.1016/j.surg.2010.03.025 